\title{
Distributed Control Design for Structures Subjected to Traveling Loads
}

\author{
Dominik Pisarski \\ Institute of Fundamental Technological Research (IPPT), Polish Academy of Sciences, Pawińskiego 5b, 02-106 Warsaw, Poland \\ Correspondence should be addressed to Dominik Pisarski; dpisar@ippt.pan.pl
}

Received 28 July 2015; Accepted 13 September 2015

Academic Editor: Qingling Zhang

Copyright ( 2015 Dominik Pisarski. This is an open access article distributed under the Creative Commons Attribution License, which permits unrestricted use, distribution, and reproduction in any medium, provided the original work is properly cited.

\begin{abstract}
This paper presents a novel distributed control method that adapts the structures subjected to traveling loads. The adaptation is realized by changes of the damping of the structure's supports. The control objective is to provide smooth passage of vehicles and to extend the safe life-time of the carrying structures. The results presented in the previous works of the author exhibited high performance of supports with an open-loop switching damping policy. In this paper, the goal is to develop a state feedback strategy that is significantly less sensitive to the system parameters and much simpler for practical implementation. Further efforts are put into designing a distributed controller architecture, where only the local and the relevant neighboring states are used to compute the control decisions. The proposed controller is validated experimentally. It exhibits high performance in a wide range of travel speeds. The practicality of the proposed solution should attract the attention of practicing engineers.
\end{abstract}

\section{Introduction}

Problems of structures subjected to loads traveling with high velocity are of special interest for practicing engineers. Numerous analytic and numerical solutions are being applied to solve the problems of transportation and robotic systems with single or multipoint interactions, such as train-track, vehicle-bridge, or effector-guideway. These problems concern the high vibration levels of both the structures and the traveling objects, due to continually increasing speeds and load carrying capacity requirements. The construction of new railway tracks or bridges with a sufficiently higher load carrying capacity and ability to withstand dynamical stresses and strains is usually limited by costs. On the other hand, a static strengthening increases the structure's mass and is often restricted for technological reasons. To face the undesired vibration effects, a variety of control systems acting on both the structures and the suspension of the traveling loads have been proposed and put into practice.

A common objective in structural control is to enhance the stability of the systems subjected to impulsive or periodic excitation. The first group of control methods, referred to as the active methods, is based on force actuators. An active control method to control the beam vibrations via linear force actuators is presented, for example, in [1]. A similar control problem, adapting a piezoelectric layer, was considered in [2]. An actively controlled beam subjected to a harmonic excitation was presented in [3]. An actively controlled string system was considered in [4]. Interesting results on the control of a cantilever beam by the use of electromagnetic actuators can be found in [5]. In the active methods in structural control we can also include the control of the track's shape. In [6], the authors developed an approach that uses active smart sleepers that enable the track to shift up and down. The objective was to minimize the deflection of the track. In [7], the authors suggested an active control method based on the linear quadratic regulator to suppress the vibration of bridges.

A recent trend is to replace force actuators with semiactive magnetorheological dampers. These solutions are usually less efficient. However, they attract engineers' interest, due to their significantly lower power consumption. They are also safer in the case of a control system failure. Unlike active systems, the semiactive ones based on controlled dampers can be always switched to passive mode, providing system stability. One of the first concepts of the semiactive control 
in mechanical systems was proposed by Karnopp, Crosby, and Harwood. In [8], they presented the idea of stabilizing of the oscillator with one degree of freedom moving upon uneven ground. The algorithm developed by the authors, "Skyhook," is today one of the most widely used ones in suspension control systems for vehicles. The idea was initially designed to improve the comfort of passengers. Later, similar control method was adopted to the oscillator moving upon carrying structures. Extensive results were demonstrated in $[9,10]$. Controlled dampers are incorporated also for seismic isolation. Interesting results can be found in $[11,12]$. In [13], the authors proposed to control both the stiffness and damping parameters. The control decision led to the maximum dissipation of energy.

The use of semiactive supports for a structure subjected to a moving load was first proposed in [14]. By means of numerical simulations, the authors demonstrated that, for a wide range of travel velocities, switching damping strategies outperform standard passive solutions. The idea was later extended in $[15,16]$, where by introducing a rigorous analysis and optimization techniques the authors concluded that even one switching action for each damper can provide very smooth load passages. A metric corresponding to the total deflection of the load trajectory from the desired straight line was reduced by up to 50 percent. The drawbacks of the proposed method lie in its complicated implementation and high sensitivity to system uncertainties. As the authors demonstrated, in order to provide a desired performance, open-loop switched solutions need to be recomputed every time we expect a different travel speed.

This paper proposes a novel control method to reduce the vibration levels of carrying structures subjected to moving loads. The method is dedicated to the applications to large scale structures like bridges and overpasses subjected to traveling trains as well as to robotic guideways subjected to effectors performing technological processes, for example, cutting, bonding, or painting. The control objective is to provide a smooth passage of the traveling load and to extend the safe life-time of the carrying structures. The proposed control strategy is based on the optimal centralized openloop solutions presented in $[15,16]$, but due to a distributed controller architecture and a state feedback control law, it is a lot more practical and robust.

For the distributed controller, we will impose the following requirements: functional symmetry in the controller's structure, the minimum computational burden, and information exchange. The symmetry will be achieved by splitting the controller into modules realizing the same computational procedures. The modular type of architecture is convenient for system assembly and maintenance. Each of the modules will compute its control decision by using local state information and the average state of the whole structure. The average state will be reconstructed by exchanging information between the neighboring controllers. The final tuning of the controller was done by solving a corresponding optimization problem. Then, a series of experiments on a physical model of a controlled guideway was performed. As will be demonstrated, the proposed control method provides a performance

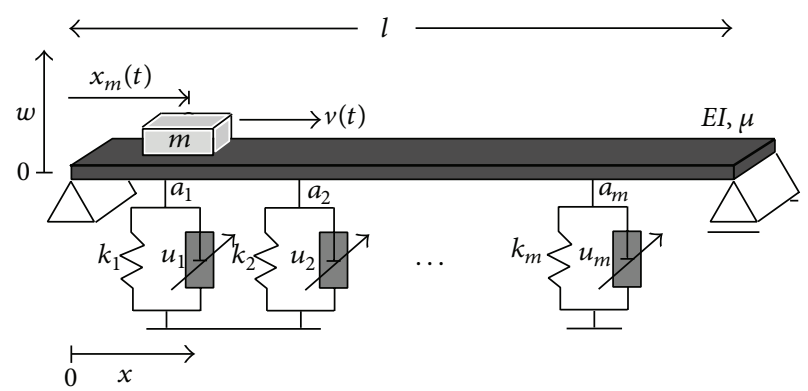

FIgURE 1: A span supported by set of controlled dampers and subjected to a mass travelling at speed $v(t)$.

that is comparable with the optimal centralized solutions, and it is robust to changes in the speed of the traveling load.

The rest of this paper is structured as follows. A mathematical model of a carrying structure supported with a set of controlled dampers and subjected to a traveling load is presented in Section 2. Section 3 introduces the distributed controller architecture and defines the local control laws. The distributed averaging problem is studied in Section 4. In Section 5, an explicit procedure for the controller implementation is given. In Section 6, a method for finding an optimal parameter of the controller is presented. Finally, the results of experimental tests of the performance of the designed controller will be presented in Section 7 .

\section{Investigated System}

In this section, we will introduce a mathematical representation of a carrying structure supported by set of controlled dampers, illustrated in Figure 1. This representation will be used in the final tuning of the proposed controller (see Section 6).

For the model of the span, we will use the Euler-Bernoulli beam equation, which is broadly applied to thin elastic bodies subjected to small deflections. A beam of the total length $l$ is characterized by the bending stiffness $E I$ and density per unit length $\mu$. A set of controlled supports are located at the positions $a_{i}$. For each of the supports, we assume a controlled damping $u_{i}$ and/or a fixed stiffness $k_{i}$. The beam is subjected to a mass $m$ travelling at the time-varying velocity $v=v(t)$ that is assumed to be given. Position of the mass is denoted by $x_{m}(t)$ and computed by using

$$
x_{m}(t)=\int_{0}^{t} v(t) d t
$$

Denoting the transverse deflection of the span by $w(x, t)$, the system is governed by

$$
\begin{aligned}
& E I \frac{\partial^{4} w(x, t)}{\partial x^{4}}+\mu \frac{\partial^{2} w(x, t)}{\partial t^{2}} \\
& =-\sum_{i=1}^{m}\left(k_{i} w\left(a_{i}, t\right)+u_{i} \frac{\partial w\left(a_{i}, t\right)}{\partial t}\right) \delta\left(x-a_{i}\right) \\
& \quad-m\left(g+\frac{\partial^{2} w\left(x_{m}(t), t\right)}{\partial t^{2}}\right) \delta\left(x-x_{m}(t)\right) .
\end{aligned}
$$


The endpoint supports impose the following boundary conditions:

$$
\begin{aligned}
w(0, t) & =0, \\
w(l, t) & =0, \\
\left(\frac{\partial^{2} w}{\partial x^{2}}\right)_{\mid x=0} & =0, \\
\left(\frac{\partial^{2} w}{\partial x^{2}}\right)_{\mid x=l} & =0 .
\end{aligned}
$$

For the initial conditions, we assume

$$
\begin{gathered}
w(x, 0)=0, \\
\dot{w}(x, 0)=0 .
\end{gathered}
$$

The left-hand side of (2) consists of the standard terms of the Euler-Bernoulli equation corresponding to the potential and inertial forces of the beam. The first two terms of the right hand side stand for the reactions of the controlled supports. The last terms correspond to the excitation of the moving load. For this excitation, we take into account both gravity and the inertial force. The latter is often ignored for large scale structures. For the systems where the masses of the span and moving load are comparable (e.g., maglev trains, robotics), the inertial force of the moving mass plays a key role in the dynamics (see $[17,18]$ ).

2.1. ODE Representation. For the sake of further studies we will now represent (2) as a set of the ordinary differential equations. Let us first introduce the orthogonal basis

$$
\theta_{\bar{j}}(x)=\sin \left(\frac{\bar{j} \pi x}{l}\right), \quad \bar{j}=1,2, \ldots
$$

It clearly fulfils the boundary conditions (3). For such a basis, a solution of (2) can be represented as follows:

$$
w(x, t)=\frac{2}{l} \sum_{\bar{j}=1}^{\infty} V_{\bar{j}}(t) \theta_{\bar{j}}(x) .
$$

By inserting (6) into (2), we obtain

$$
\begin{gathered}
\frac{2 E I}{l} \frac{\partial^{4}}{\partial x^{4}}\left(\sum_{\bar{j}=1}^{\infty} V_{\bar{j}}(t) \theta_{\bar{j}}(x)\right)+\frac{2 \mu}{l} \\
\cdot \frac{\partial^{2}}{\partial t^{2}}\left(\sum_{\bar{j}=1}^{\infty} V_{\bar{j}}(t) \theta_{\bar{j}}(x)\right)=-\frac{2}{l} \sum_{i=1}^{m} k_{i} \\
\cdot \sum_{\bar{j}=1}^{\infty} V_{\bar{j}}(t) \theta_{\bar{j}}\left(a_{i}\right) \delta\left(x-a_{i}\right)-\frac{2}{l}
\end{gathered}
$$

$$
\begin{aligned}
& \cdot \sum_{i=1}^{m} u_{i} \frac{\partial}{\partial t}\left(\sum_{\bar{j}=1}^{\infty} V_{\bar{j}}(t) \theta_{\bar{j}}\left(a_{i}\right)\right) \delta\left(x-a_{i}\right) \\
& -m\left[g+\frac{\partial^{2}}{\partial t^{2}}\left(\frac{2}{l} \sum_{\bar{j}=1}^{\infty} V_{\bar{j}}(t) \theta_{\bar{j}}\left(x_{m}(t)\right)\right)\right] \\
& \cdot \delta\left(x-x_{m}(t)\right) .
\end{aligned}
$$

Now each term of (7) is multiplied by

$$
\theta_{j}(x)=\sin \left(\frac{j \pi x}{l}\right)
$$

and then integrated with respect to $x$ over the interval $[0, l]$. This results in the following weak formulation:

$$
\begin{aligned}
& \frac{2}{l} \sum_{\bar{j}=1}^{\infty}\left[E I\left(\frac{\bar{j} \pi}{l}\right)^{4} V_{\bar{j}}(t)+\mu \ddot{V}_{\bar{j}}(t)\right] \int_{0}^{l} \theta_{\bar{j}}(x) \theta_{j}(x) d x \\
& =-\frac{2}{l} \sum_{i=1}^{m} \sum_{j=1}^{\infty} k_{i} V_{\bar{j}}(t) \int_{0}^{l} \theta_{\bar{j}}\left(a_{i}\right) \theta_{j}(x) \delta\left(x-a_{i}\right) d x \\
& -\frac{2}{l} \sum_{i=1}^{m} \sum_{j=1}^{\infty} u_{i} \dot{V}_{\bar{j}}(t) \int_{0}^{l} \theta_{\bar{j}}\left(a_{i}\right) \theta_{j}(x) \delta\left(x-a_{i}\right) d x \\
& -m_{g} \int_{0}^{l} \theta_{j}(x) \delta\left(x-x_{m}(t)\right) d x-\frac{2 m}{l} \\
& \cdot \frac{\partial^{2}}{\partial t^{2}}\left(\sum_{\bar{j}=1}^{\infty} V_{\bar{j}}(t) \theta_{\bar{j}}\left(x_{m}(t)\right)\right) \\
& \cdot \int_{0}^{l} \theta_{j}(x) \delta\left(x-x_{m}(t)\right) d x .
\end{aligned}
$$

Note that (9) must hold for every $j=1,2, \ldots$.. Now we can use the orthogonality condition for the $\theta$ functions; that is,

$$
\int_{0}^{l} \theta_{\bar{j}}(x) \theta_{j}(x) d x=\frac{l}{2} \delta_{\bar{j}, j} .
$$

Here, $\delta_{\bar{j}, j}$ stands for the Kronecker delta. By using the sifting property of the Dirac delta function, we can also give explicit formulas for the terms:

$$
\begin{gathered}
\int_{0}^{l} \theta_{\bar{j}}\left(a_{i}\right) \theta_{j}(x) \delta\left(x-a_{i}\right) d x=\theta_{\bar{j}}\left(a_{i}\right) \theta_{j}\left(a_{i}\right), \\
\int_{0}^{l} \theta_{j}(x) \delta\left(x-x_{m}(t)\right)=\theta_{j}\left(x_{m}(t)\right) .
\end{gathered}
$$


Thus, (9) can be written as follows:

$$
\begin{aligned}
\mu \sum_{\bar{j}=1}^{\infty} \ddot{V}_{\bar{j}}(t) \delta_{\bar{j}, j}+E I \sum_{\bar{j}=1}^{\infty}\left(\frac{\bar{j} \pi}{l}\right)^{4} V_{\bar{j}}(t) \delta_{\bar{j}, j} \\
=-\frac{2}{l} \sum_{i=1}^{m} \sum_{\bar{j}=1}^{\infty}\left(k_{i} V_{\bar{j}}(t)+u_{i} \dot{V}_{\bar{j}}(t)\right) \theta_{\bar{j}}\left(a_{i}\right) \theta_{j}\left(a_{i}\right) \\
\quad-m g \theta_{j}\left(x_{m}(t)\right) \\
\quad-\frac{2 m}{l} \frac{\partial^{2}}{\partial t^{2}}\left(\sum_{\bar{j}=1}^{\infty} V_{\bar{j}}(t) \theta_{\bar{j}}\left(x_{m}(t)\right)\right) \theta_{j}\left(x_{m}(t)\right), \\
j=1,2, \ldots
\end{aligned}
$$

It still remains to compute the second time derivative of $\sum_{\bar{j}=1}^{\infty} V_{\bar{j}}(t) \theta_{\bar{j}}\left(x_{m}(t)\right)$. An explicit formula for this derivative is given below:

$$
\begin{aligned}
\frac{\partial^{2}}{\partial t^{2}}\left(\sum_{\bar{j}=1}^{\infty} V_{\bar{j}}(t) \theta_{\bar{j}}\left(x_{m}(t)\right)\right) \\
=\sum_{\bar{j}=1}^{\infty} \ddot{V}_{\bar{j}}(t) \theta_{\bar{j}}\left(x_{m}(t)\right) \\
\quad+\sum_{\bar{j}=1}^{\infty} \frac{2 \bar{j} \pi}{l} \dot{V}_{\bar{j}}(t) \phi_{\bar{j}}\left(x_{m}(t)\right) v(t) \\
\quad+\sum_{\bar{j}=1}^{\infty} \frac{\bar{j} \pi}{l} V_{\bar{j}}(t) \phi_{\bar{j}}\left(x_{m}(t)\right) \dot{v}(t) \\
\quad-\sum_{\bar{j}=1}^{\infty} \frac{\bar{j}^{2} \pi^{2}}{l^{2}} V_{\bar{j}}(t) \theta_{\bar{j}}\left(x_{m}(t)\right)(v(t))^{2} .
\end{aligned}
$$

Here, we use the notation $\phi_{\bar{j}}\left(x_{m}(t)\right)=\cos \left(\bar{j} \pi x_{m}(t) / l\right)$. Regarding only these terms in (12) where the Kronecker deltas are not equal to zero, we can finally rewrite (2) as follows:

$$
\begin{aligned}
& w(x, t)=\frac{2}{l} \sum_{\bar{j}=1}^{\infty} V_{\bar{j}}(t) \theta_{\bar{j}}(x), \\
& \mu \ddot{V}_{j}(t)+E I\left(\frac{j \pi}{l}\right)^{4} V_{j}(t) \\
& =-\frac{2}{l} \sum_{i=1}^{m} \sum_{j=1}^{\infty}\left(k_{i} V_{\bar{j}}(t)+u_{i} \dot{V}_{\bar{j}}(t)\right) \theta_{\bar{j}}\left(a_{i}\right) \theta_{j}\left(a_{i}\right) \\
& -m g \theta_{j}\left(x_{m}(t)\right) \\
& \quad-\frac{2 m}{l} \sum_{\bar{j}=1}^{\infty} \ddot{V}_{\bar{j}}(t) \theta_{\bar{j}}\left(x_{m}(t)\right) \theta_{j}\left(x_{m}(t)\right) \\
& -\frac{4 m}{l} \sum_{\bar{j}=1}^{\infty} \frac{\bar{j}^{j} \pi}{l} \dot{V}_{\bar{j}}(t) \phi_{\bar{j}}\left(x_{m}(t)\right) \theta_{j}\left(x_{m}(t)\right) v(t)
\end{aligned}
$$

$$
\begin{array}{r}
-\frac{2 m}{l} \sum_{\bar{j}=1}^{\infty} \frac{\bar{j} \pi}{l} V_{\bar{j}}(t) \phi_{\bar{j}}\left(x_{m}(t)\right) \theta_{j}\left(x_{m}(t)\right) \dot{v}(t) \\
+\frac{2 m}{l} \sum_{\bar{j}=1}^{\infty} \frac{\bar{j}^{2} \pi^{2}}{l^{2}} V_{\bar{j}}(t) \theta_{\bar{j}}\left(x_{m}(t)\right) \theta_{j}\left(x_{m}(t)\right)(v(t))^{2}, \\
j=1,2, \ldots .
\end{array}
$$

The initial condition is now given by

$$
\begin{aligned}
& V_{j}(0)=0, \\
& \dot{V}_{j}(0)=0,
\end{aligned}
$$

$$
j=1,2, \ldots
$$

2.2. State-Space Representation, Control Bounds. From (14) we can observe that, at each time instant, the state of the system is fully characterized by the sets $\left\{V_{j}(t)\right\}$ and $\left\{\dot{V}_{j}(t)\right\}$. Throughout the rest of the paper, we will rely on an approximated representation of (14) by taking $j$ from 1 to $n=10$. The assumed ten modes are sufficient to render the dynamics of span while keeping the size of the system eligible for efficient optimization.

By introducing the state vector $y \in \mathbb{R}^{2 n}$ such that

$$
\begin{aligned}
y(t) & \\
& =\left[V_{1}(t), \dot{V}_{1}(t), V_{2}(t), \dot{V}_{2}(t), \ldots, V_{n}(t), \dot{V}_{n}(t)\right]^{T},
\end{aligned}
$$

we can represent (14) by the following time-varying bilinear system:

$$
\begin{aligned}
\dot{y} & =A(t) y+\sum_{i=1}^{m} u_{i} B_{i}(t) y+F(t), \\
y(0) & =0 .
\end{aligned}
$$

Here, the matrices $A$ and $B$ are mainly built upon the eigenfunctions $\phi$ and $\theta$, while the excitation vector $F$ collects the terms related to the force of gravity (for detailed structures see [19]). For the controls, we assume that each one is bounded by two positive values corresponding to the minimum and maximum admissible damping coefficients; that is,

$$
u \in \mathcal{U}=\left[u_{\min }, u_{\max }\right]^{m} \subset \mathbb{R}_{+}^{m} .
$$

\section{Control Design}

The goal of this section is to introduce a distributed state feedback control law for system (17). First, let us characterize the distributed controller architecture.

3.1. Distributed Controller Architecture. For each of the controlled supports, we will now associate a local state as illustrated in Figure 2. The control decision $u_{i}$, together with 


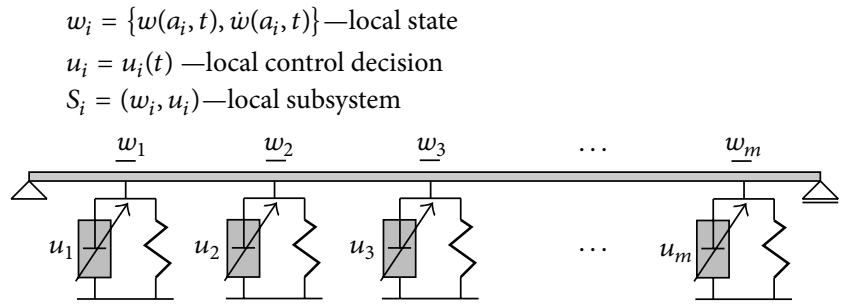

FIGURE 2: A controlled beam represented as a set of local subsystems.

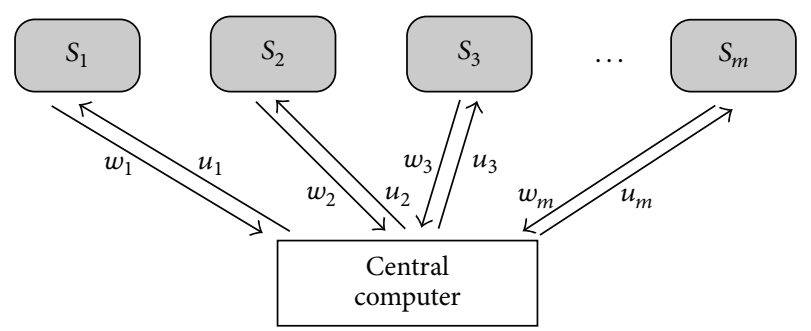

FIGURE 3: Centralized controller architecture. The whole system is managed by a central computer.

the associated state $w_{i}=\left\{w\left(a_{i}, t\right), \dot{w}\left(a_{i}, t\right)\right\}$, will be referred to as the subsystem and be denoted by $S_{i}$.

For such representation, a centralized controller architecture can be visualized as shown in Figure 3. All accessible states are first transferred to a central computing unit. Based on the given information, the unit takes a decision that is sent to all control devices. In a distributed architecture, we assume that each of the local subsystems is managed by its own computing unit, as depicted in Figure 4 . That, practically, means that every local computer is allowed to measure only its local state and send a control signal to a corresponding local control device. Note that, due to the coupled dynamics, each of the local decisions affects the whole system. Thus, a controller relying only on the local states may lead to undesired states. To improve performance and to provide robustness, a distributed controller allows local computers to exchange some information. In our setting, we assume that computers 1 to $m-1$ send their state information to their right neighbors, while computer $m$ delivers the state to computer 1 . The assumed communication topology will be later used for solving the distributed averaging problem.

The proposed distributed architecture is modular and has functional symmetry; that is, each of the local controllers can be seen as a module realizing the same procedure. This type of architecture is convenient for system assembly and maintenance, in particular for large scale structures. In the case of failure, only the malfunctioning module needs to be replaced. In addition, such a modular decentralization plays an important role in safety. Suppose there is a malfunction of the central computer. An incorrect signal is then sent to the whole system. In a modular controller architecture, this risk is reduced to local failures. For an executive component of a proposed module, one can consider a rotary magnetorheological damper equipped with an encoder (see http://www.lord.com/).

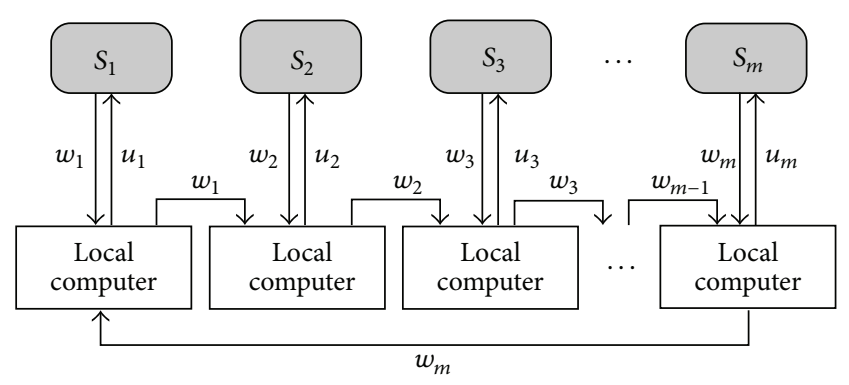

FIgURE 4: Distributed controller architecture. The system is operated by a set of local computers allowed to exchange state information.

3.2. Distributed Control Law. Under the assumed distributed architecture, we will now design a control law to be performed by each of the local controllers. The first requirement is that this control law is based on the accessible state information and can be implemented in real-time. We also require that the resulting closed-loop system is robust in the sense that it is not sensitive to small changes of the system parameters, in particular changes in the velocity of the moving load. This is of special importance for structures subjected to vehicles for which we can naturally expect various speeds. The common control objective is to reduce the vibration levels of the structure, as well as to provide a smooth straight line passage for the traveling load.

The control law introduced in this work is heuristic. However, it arises from the analysis on the system dynamics as well as the optimal solutions studied in $[15,16,19,20]$. The idea of a straight line passage is based on the fundamental principle of a two-sided lever. In Figure 5, we illustrate this idea for a beam supported by two controlled dampers. During the first stage of a passage, for the left damper we set its maximum admissible damping value providing that the deflection under a moving load maintains moderate levels. At the same time, the damper on the right operates at its minimum value enabling a beam to turn around its center of gravity and to lever its right hand part. This temporal increment of displacement of the right hand part results in a nearly straight line load trajectory during the second stage of the passage, when the operation modes of the dampers are reversed.

Formally, the problem of an optimally straight line passage for system (17) can be written as follows:

$$
\text { find } \begin{aligned}
u^{*}=\underset{u \in \mathscr{U}}{\operatorname{argmin}} J & =\int_{0}^{T} y^{T} Q(t) y d t \\
& \text { under } \dot{y}=A(t) y+\sum_{i=1}^{m} u_{i} B_{i}(t) y+F(t) .
\end{aligned}
$$

Here, the time horizon $T$ is equal to the total time of a passage and is computed by using

$$
l=\int_{0}^{T} v(t) d t .
$$

The matrix $Q(t)$ is taken such that

$$
y^{T} Q(t) y=(w(v t, t))^{2} .
$$



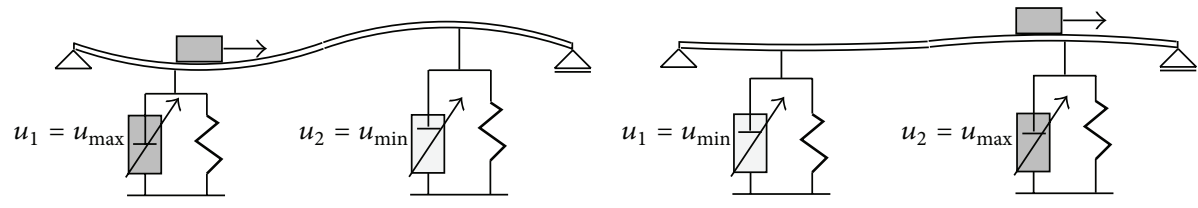

FIGURE 5: The idea of a straight line passage based on the principle of a two-sided lever.

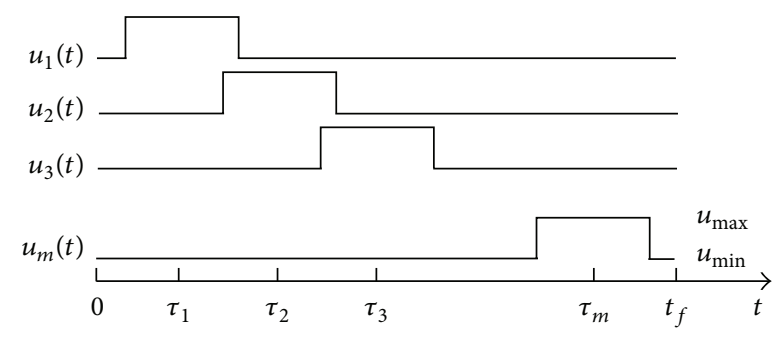

FIGURE 6: Diagonal structure of the optimal controls for straight line passage of a moving load.

Thus, the objective function corresponds to the total deflection of the trajectory of a moving load.

A comprehensive study of a solution to problem (19) was presented in [19]. The author demonstrated that, for a wide range of system parameters, the solutions exhibit similar structures. This structure is represented by the diagonal pattern depicted in Figure 6. Here, $\tau_{i}$ stands for the time instant in which a traveling load is passing the position $a_{i}$. The practical meaning of this pattern is that each damper is supposed to be switched to the maximum admissible value just before the load approaches it, while the switch back to the minimum value is supposed to be done right after that passage. In this paper, we will design a state feedback control law that mimics the optimal diagonal pattern, providing also robustness to a wide range of travel speed. The key in deriving this control law is the observation of how the state of a beam corresponds to the horizontal position of a moving load.

Suppose that for the beam presented in Figure 2 we put two observers. The first one focuses on the transverse velocity of the point $a_{1}$, that is, $\dot{w}\left(a_{1}, t\right)$, while the other collects the transverse velocities for all points $a_{i}$ and computes the average of the absolute values: $(1 / m) \sum_{i=1}^{m}\left|\dot{w}\left(a_{i}, t\right)\right|$. On the left hand side of Figure 7 , an approximate evolution of $\dot{w}\left(a_{1}, t\right)$ during the passage is depicted. We can observe that the local transverse velocity reaches its highest negative values when the moving load is in the neighborhood of the position $a_{1}$. Then, it slowly changes sign and $a_{1}$ returns toward the equilibrium. A similar behavior can be observed over a wide range of passage speeds, with the tendency that a larger speed magnifies the trajectory. (If the local observer is located at the position $a_{i}$, then the trajectory is analogous except for the convex part that is shifted to $\tau_{i}$.) The second observer notices that the average $(1 / m) \sum_{i=1}^{m}\left|\dot{w}\left(a_{i}, t\right)\right|$ rapidly increases and remains at some level up to the end of the passage (see the right-hand side of Figure 7). A similar result is also valid for different passage velocities, with the tendency that larger speeds magnify the trajectory. Our task now is to reconstruct the desired control action, denoted by the dotted lines, with the use of the measurements by both observers. The reader can verify that this action can be approximately provided by the following control law:

$$
u_{i}(t)= \begin{cases}u_{\max }, & \text { if } \dot{w}\left(a_{i}, t\right) \leq-\alpha \frac{1}{m} \sum_{i=1}^{m}\left|\dot{w}\left(a_{i}, t\right)\right|, \\ u_{\min }, & \text { otherwise. }\end{cases}
$$

Here $\alpha>0$. The control law states that we are supposed to set the maximum control value only when the local transverse velocity is below the bound given by the average absolute value (more precisely, some portion of this average, where the portion is determined by the constant parameter $\alpha$ ). The role of the average is to adapt the switching bound to different passage speeds. From Figure 7 one can notice that if we relied on some fixed switching bound, then the controls may operate on the maximum values, either too shortly (for slow passages) or too long (in case of fast travels).

The controller given by (22) is supposed to be implemented with the information allowed by the distributed architecture (Figure 4). While the local state is assumed to be given, the average needs to be computed in a distributed manner with the use of the circular information exchange between the local computers. The problem of distributed averaging will be studied in the next section. The method for selecting the parameter $\alpha$ will be presented in Section 6 .

\section{Distributed Averaging Problem}

The controller given by (22) is supposed to be implemented with the information allowed by the distributed architecture (Figure 4). While the local state is assumed to be given, the average needs to be computed in a distributed manner with the use of the circular information exchange between the local computers. Before we give an algorithm for distributed averaging, let us first introduce a relevant background. We will now consider a linear dynamical system

$$
\begin{gathered}
z(k+1)=A z(k), \quad k=0,1, \ldots, \\
z(0)=z_{0}, \quad z \in \mathbb{R}^{n} .
\end{gathered}
$$

The distributed averaging problem is formulated as follows.

Find the matrix $A$ such that the state of system (23) converges to a common value that is equal to the average of the initial state.

A system solving the distributed averaging problem is a particular case of the consensus protocols. A comprehensive theoretical framework for consensus problems can be found 


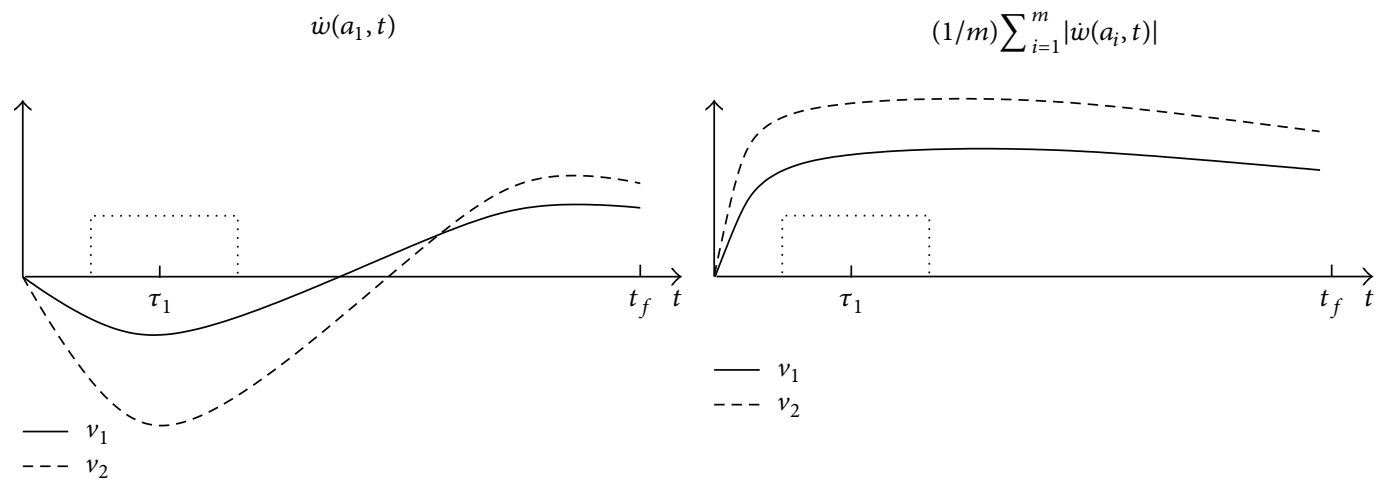

Figure 7: Evolution of the local transverse velocity $\dot{w}\left(a_{1}, t\right)$ and the average of the absolute values of the local transverse velocities $(1 / m) \sum_{i=1}^{m}\left|\dot{w}\left(a_{i}, t\right)\right|$ for two different moving load speeds $v_{1}<v_{2}$. Dotted lines mark the desired shape for control $u_{1}$.

in [21]. Here, we will present only the fundamental results for distributed averaging under linear dynamics.

Let $c$ be a real number. We say that system (23) solves the consensus problem (or that system (23) is a consensus protocol) if and only if there exists an asymptotically stable equilibrium $z^{*}$ satisfying

$$
z^{*}=c \mathbf{1},
$$

where 1 stands for the all-ones vector. A necessary and sufficient condition for system (23) to be a consensus protocol is that $A$ has 1 as a simple eigenvalue, with the corresponding eigenvector having equal components, and all other eigenvalues are located inside the unit circle. Of special interest regarding these spectral properties is the family of matrices called stochastic matrices, that is, square matrices of nonnegative real numbers, with each row summing to 1 . It follows from the definition that for a stochastic $A$ we have

$$
A 1=1 .
$$

Therefore, a stochastic $A$ always has an eigenvalue of 1 with the corresponding eigenvector $\mathbf{1}$. To verify that the other eigenvalues of a stochastic $A$ are inside the unit circle, one can follow the Gershgorin theorem [22]. To guarantee that system (23) solves the consensus problem, it is required that $A$ has 1 as a simple eigenvalue. This property can be verified by checking the connectivity of the corresponding directed graph. (It is required that the connectivity graph has a spanning tree.)

As stated in the distributed averaging problem, we look for a structure of $A$ that provides that system (23) converges to a common value, but in addition, this common value is the average of the components of the initial state vector. To fulfill the last requirement, we impose an additional condition on A:

$$
A^{T} \mathbf{1}=\mathbf{1},
$$

meaning that each column of $A$ sums to 1 . A matrix with nonnegative real entries for which (25) and (26) hold is called doubly stochastic. It can be proven (see, e.g., [23]) that, for a doubly stochastic matrix $A$,

$$
\lim _{k \rightarrow \infty} A^{k}=\frac{1}{n} \mathbf{1 1}^{T} \text {. }
$$

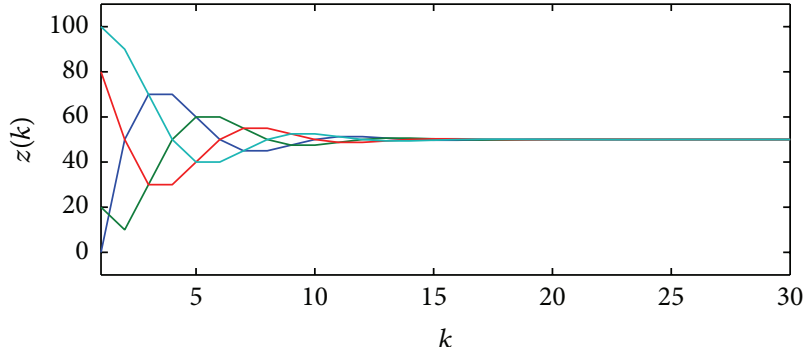

FIGURE 8: Evolution of the dynamics of the consensus protocol (29). The common value is the average of the entries of the initial state.

Thus, system (23) with a doubly stochastic $A$ has an asymptotically stable equilibrium

$$
z^{*}=\frac{\sum_{i=1}^{n} z_{i}(0)}{n} \mathbf{1}
$$

An example of a system solving the average consensus problem is

$$
z(k+1)=\left[\begin{array}{cccc}
0.5 & 0 & 0 & 0.5 \\
0.5 & 0.5 & 0 & 0 \\
0 & 0.5 & 0.5 & 0 \\
0 & 0 & 0.5 & 0.5
\end{array}\right] z(k)
$$

The dynamics of protocol (29) is illustrated in Figure 8.

\section{Implementation of the Distributed Controller}

Now we will focus on how to apply a consensus protocol for our distributed controller. The assumption made here is that we take the control decision given by (22) only once per time needed to execute a consensus protocol. Current technology enables executing 10 iterations of a consensus protocol for several neighboring agents within less than 50 milliseconds 
(this time includes the required communication with the use of Fast Ethernet).

The idea of the distributed computing of $(1 / m) \sum_{i=1}^{m}\left|\dot{w}\left(a_{i}, t\right)\right|$ is as follows. At time $t$, each of the local controllers measures its local transverse velocity $\dot{w}\left(a_{i}, t\right)$. Then, each of the controllers makes the assignment

$$
z_{0}^{i}=\left|\dot{w}\left(a_{i}, t\right)\right|
$$

This makes up the initial vector

$$
z_{0}=\left[z_{0}^{1}, z_{0}^{2}, \ldots, z_{0}^{m}\right]^{T}
$$

By using the circular communication channels depicted in Figure 4 the controllers exchange the values of $z$ and perform a consensus protocol:

$$
z(k+1)=A z(k), \quad k=0,1, \ldots, z(0)=z_{0}
$$

Here $A_{m \times m}$ is doubly stochastic and structured as follows:

$$
A=\left[\begin{array}{cccc}
1-h & 0 & 0 & h \\
h & 1-h & 0 & 0 \\
0 & \ddots & \ddots & 0 \\
0 & 0 & h & 1-h
\end{array}\right], \quad h \in(0,1)
$$

After a certain number $k_{t m}$ of iterations, the protocol is terminated and each of the controllers makes the assignment

$$
\frac{1}{m} \sum_{i=1}^{m}\left|\dot{w}\left(a_{i}, t\right)\right|=z^{i}\left(k_{t m}\right)
$$

Note that the average recovered by (34) may slightly differ for local controllers. To provide more accurate results, one can consider an optimization problem to find the optimal $h$ in (33) resulting in the best convergence rates for the consensus protocol. This goes beyond the scope of this paper and is reserved for future research. For the numerical and experimental studies, we selected $h=0.5$.

From the perspective of a local controller $i$, at every decision time $t$, the following procedure is supposed to be executed.

\section{Local Controller Procedure}

Step 1. Initialize $k=0$.

Step 2. Measure $\dot{w}\left(a_{i}, t\right)$ and assign $z^{i}(k)=\left|\dot{w}\left(a_{i}, t\right)\right|$.

Step 3. Send $z^{i}(k)$ to controller $i+1$ (if $i=m$, then send $z^{m}(k)$ to controller 1).

Step 4. $\operatorname{Read} z^{i-1}(k)$ (if $i=1$, then $\operatorname{read} z^{m}(k)$ ).

Step 5. Update: $z^{i}(k+1)=(1-h) z^{i}(k)+h z^{i-1}(k)$ (if $i=1$, then update $\left.z^{1}(k+1)=(1-h) z^{1}(k)+h z^{m}(k)\right)$.

Step 6. Update: $k=k+1$.

Step 7. If $k \leq k_{t m}$, then go to Step 3; else perform the control:

$$
u_{i}(t)= \begin{cases}u_{\max }, & \text { if } \dot{w}\left(a_{i}, t\right) \leq-\alpha z^{i}(k), \\ u_{\min }, & \text { otherwise. }\end{cases}
$$

\section{Selection of the Control Law Parameter " $\alpha$ "}

For the parameter $\alpha$ (see the distributed control law (22)) we are interested in the selection providing that our distributed controller mimics the behavior of the optimal open-loop controller generated by the solution of (19). In the formulation of the corresponding optimization problem, we will use the state-space representation introduced in Section 2.2.

Let $y_{j}$ denote the $j$ th component of the state vector defined in (16). Now, according to (6) (with the first ten modes taken into account) the transverse displacement and velocity can be, respectively, approximated by

$$
\begin{aligned}
& w(x, t)=\frac{2}{l} \sum_{j=1}^{10} y_{2 j-1} \sin \left(\frac{j \pi x}{l}\right), \\
& \dot{w}(x, t)=\frac{2}{l} \sum_{j=1}^{10} y_{2 j}(t) \sin \left(\frac{j \pi x}{l}\right) .
\end{aligned}
$$

We consider again the matrix $Q(t)$ defined as in (21). We look for $\alpha$ that is the solution of the following problem:

$$
\text { find } \begin{aligned}
\alpha^{*}=\underset{\alpha>0}{\operatorname{argmin}} \quad J & =\int_{0}^{T} y^{T} Q(t) y d t \\
\text { subject to } \quad \dot{y} & =A(t) y+\sum_{i=1}^{m} u_{i} B_{i}(t) y+F(t), \\
u_{i} & = \begin{cases}u_{\max }, & \text { if } \sum_{j=1}^{10} y_{2 j} \sin \left(\frac{j \pi a_{i}}{l}\right) \leq-\alpha \frac{1}{m} \sum_{i=1}^{m}\left|\sum_{j=1}^{10} y_{2 j} \sin \left(\frac{j \pi a_{i}}{l}\right)\right|, \\
u_{\min }, & \text { otherwise. }\end{cases}
\end{aligned}
$$




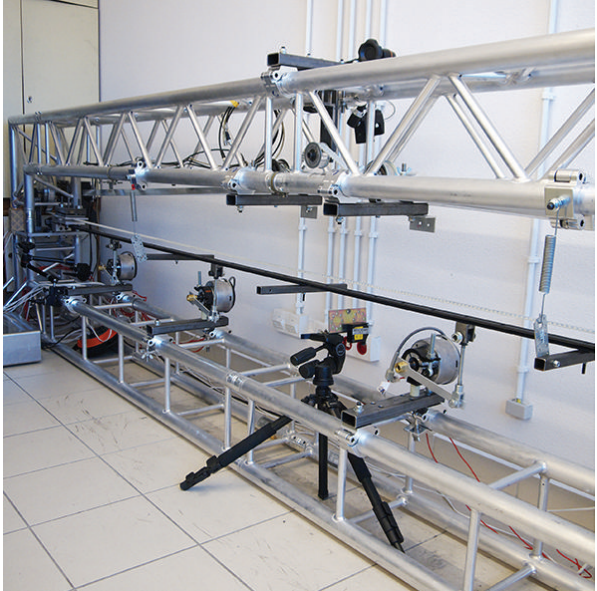

(a)

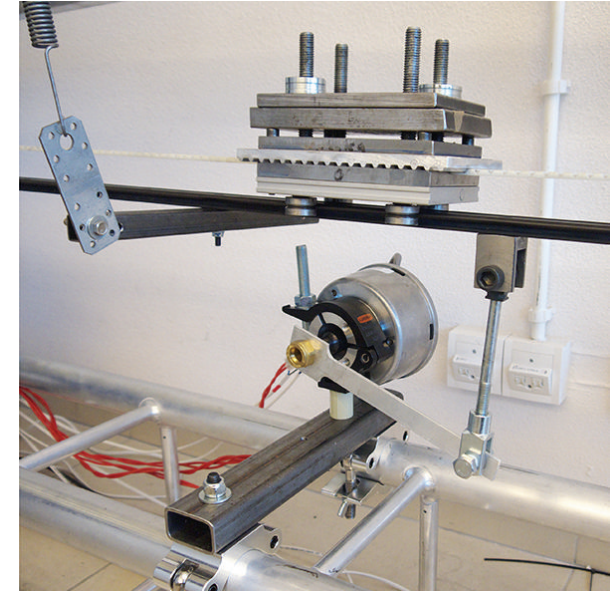

(b)

FIGURE 9: Real view of the test stand (a) and the rotary controlled damper supporting the guideway (b).

Due to the switched structure of the constraints, problem (37) was solved fully numerically. The gradient of the objective function was approximated by the finite difference scheme, and by using the second-order Runge-Kutta method the dynamics was represented by set of equations. With such representation the problem was solved with the use of Ipopt, a software package for large scale optimization (see https://projects.coin-or.org/Ipopt).

In the computations, all model parameters were taken accordingly to the experimental stand described in the next section. For the mass traveling at a speed of $1 \mathrm{~m} / \mathrm{s}, 2 \mathrm{~m} / \mathrm{s}$, and $4 \mathrm{~m} / \mathrm{s}$, the solution to (37) was $\alpha=0.694, \alpha=0.761$, and $\alpha=0.712$, respectively. In the experiments, we will rely on the average of these values; that is, $\alpha=0.722$.

\section{Experimental Validation}

In this section, the designed control method will be examined by means of the experimental results. We will be interested in both the performance and the robustness of the closedloop system. For that purpose, we will evaluate four different metrics corresponding to the state of the carrying structure and the trajectory of the moving load. In terms of these metrics, we will compare the closed-loop system with the optimal centralized open-loop solutions. In addition, we will provide a comparison to a passively damped system. To examine the robustness, we will repeat the experiments with different passage speeds.

7.1. The Test Stand. The supporting structure was made of an aluminum truss frame. The carrying structure is represented by a guideway supported with two springs to reduce the static deflection. For the traveling load, we use a carriage driven by an electric motor. For the controlled devices we use four magnetorheological rotary dampers equipped with encoders. The locations for the dampers are $a_{1}=1 / 5 l, a_{2}=2 / 5 l$, $a_{3}=3 / 5 l$, and $a_{4}=4 / 5 l$ (see Figure 9). During the passage,
TABLE 1: Parameters of the test stand.

\begin{tabular}{lc}
\hline Guideway length $(l)$ & $4 \mathrm{~m}$ \\
Guideway stiffness $(E I)$ & $801 \mathrm{Nm}^{2}$ \\
Damping range $\left(u_{\min }-u_{\max }\right)$ & $100-1300 \mathrm{Ns} / \mathrm{m}$ \\
Spring stiffness $\left(k_{1}, k_{2}\right)$ & $1000 \mathrm{~N} / \mathrm{m}$ \\
Mass of carriage $(m)$ & $0.7-10 \mathrm{~kg}$ \\
Maximum motor torque & $21 \mathrm{Nm}$ \\
Maximum speed of carriage $(v)$ & $6 \mathrm{~m} / \mathrm{s}$ \\
Maximum acceleration of carriage & $7 \mathrm{~m} / \mathrm{s}^{2}$ \\
\hline
\end{tabular}

the carriage is first rapidly accelerated from zero to a given speed. This speed is kept constant up to the last stage of the passage (corresponding to approximately $4 / 5$ of the length of the guideway), when the motor starts its braking process. For both the acceleration and the deceleration, we use the maximum admissible values, to arrange that the carriage travels at a constant speed for most of the time.

In order to design the parameters for the test stand, several numerical simulations were first performed. Custom software simulated the dynamic interactions between the traveling carriage and the whole structure. Various combinations of the support rails and guideways were taken into account. For the notable deflection of the beam, the pivotally mounted guideway without the supporting rail was selected. This resulted in the maximum transverse displacement within the range of $\pm 30 \mathrm{~mm}$ for a mass of $5 \mathrm{~kg}$ traveling at a speed of $4 \mathrm{~m} / \mathrm{s}$. The carriage was attached to the guideway with the use of a special type of conical rollers protected from jamming in the case of traveling over large deflections. The carriage was driven by a stepper motor via a toothed belt girded over a gearbox. Our setup enables accelerating a mass of $6 \mathrm{~kg}$ to the speed of $5 \mathrm{~m} / \mathrm{s}$ and stopping it within the guideway's length, which was $4 \mathrm{~m}$. The set of major parameters for the test stand is given in Table 1. 
The control system operates as follows. The state (the local transverse deflections) is first measured by the encoders incorporated into the controlled dampers. The signals are transferred to a PC equipped with an I/O data acquisition card. A custom program (written in the MATLAB language) then processes the signals to recover the absolute values of the local transverse velocities. Next, a process of distributed averaging is executed. Here, on a single PC, we simulated a distributed architecture, where each of the local controllers, represented by separate MATLAB functions communicating under the assumed information topology, executes the Local Controller Procedure given in Section 5. The procedure was terminated after $k_{t m}=10$ cycles and the control decision based on (22) was then sent to the dampers. The whole process was triggered by the motor encoder (indicating the start of the passage) and was repeated every 50 milliseconds until the end of the passage. The repeating time was selected according to the estimated time required for the distributed averaging process to be carried out in a real modular architecture. This time also corresponds to the response time of the dampers.

7.2. Experimental Results. The experiments were performed under the following parameters: mass of the carriage $m=$ $4 \mathrm{~kg}$ and passage speeds $v=1, v=2$, and $v=4 \mathrm{~m} / \mathrm{s}$. The performance of the control method will be examined in terms of the following metrics:

$$
\begin{aligned}
& J_{1}=\int_{0}^{l} \int_{0}^{T}(w(x, t))^{2} d x d t, \\
& J_{2}=\max _{x \in[0, l], t \in[0, T]}|w(x, t)|, \\
& J_{3}=\int_{0}^{T}(w(v t, t))^{2} d t, \\
& J_{4}=\max _{t \in[0, T]}|w(v t, t)| .
\end{aligned}
$$

Here $J_{1}$ and $J_{2}$ stand for the total and the maximum beam deflection, respectively. Similarly, $J_{3}$ and $J_{4}$ correspond to the total and the maximum deflection on the trajectory of the carriage. Despite the experiments having exhibited good repetitiveness (a maximum difference of 4 percent for the assumed metrics), each of the passages was repeated five times, and the values of the metrics were averaged. To reconstruct the trajectories $w(x, t)$ and $w(v t, t)$, we rely on the first four terms of series (6) (four is the number of local measurements $w\left(a_{i}, t\right)$ provided by the encoders incorporated in the dampers).

For each of the passages, three damping strategies were applied. The first one, referred to later as passive, was under a constant damping, set to the maximum admissible value $u_{\max }$. The second strategy was based on the optimal openloop solutions (referred to as centralized). These solutions were obtained by using the method of optimal switching times, described in [19], under the assumption that each of the controls can be switched at most twice. Finally, the proposed distributed strategy was performed.

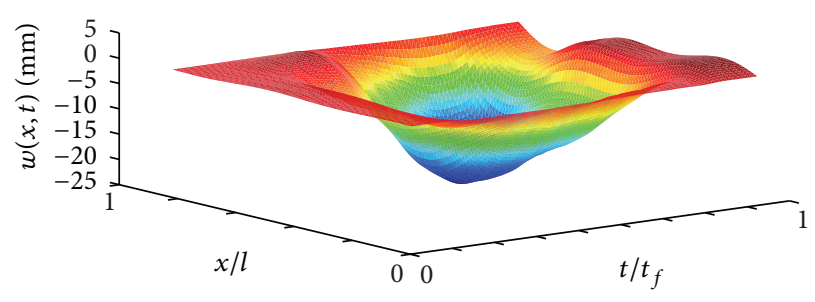

FIGURE 10: Guideway deflection in space and time in the case of $v=$ $4 \mathrm{~m} / \mathrm{s}$ under the designed distributed controller.

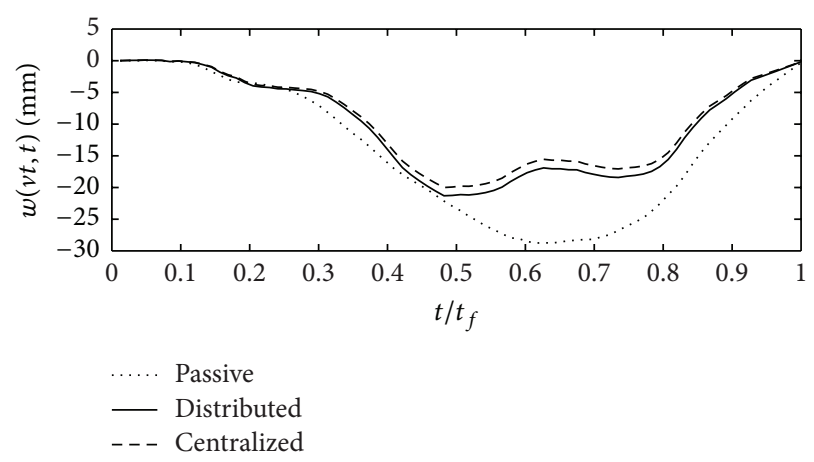

FIgURE 11: Moving load trajectories in the case of $v=4 \mathrm{~m} / \mathrm{s}$.

Typical dynamics of a carrying structure subjected to a moving load is illustrated in Figure 10. In this example, the passage at speed $v=4 \mathrm{~m} / \mathrm{s}$ was controlled by the distributed strategy. As intuitively predicted, the maximum deflection takes place approximately at $x \approx 0.5 l$, the midpoint of the guideway, that is, at $t \approx 0.5 T$. Note that the carriage is traveling on the diagonal stretched between the points $(0,0)$ and $(l, T)$.

The moving load trajectories in the case of $v=4 \mathrm{~m} / \mathrm{s}$ are compared in Figure 11. Under passive damping, the maximum deflection exceeds $35 \mathrm{~mm}$. Under the optimal centralized control, this deflection is reduced by 34 percent. The distributed method results in an improvement by 29 percent. Both controlled trajectories exhibit analogous shapes, which is due to their similar control functions; see Figures 12 and 13 for the centralized and distributed controls, respectively. We can observe that only the first control differs in the number of switches. The diagonal pattern of the optimal controls is well reproduced by the distributed method, with slight shifts of the switching times. An analogy in the control shapes was also observed for the passages at speeds $v=1$ and $v=2 \mathrm{~m} / \mathrm{s}$.

A comparison of moving load trajectories in the case of $v=2 \mathrm{~m} / \mathrm{s}$ is presented in Figure 14. Again, both controlled strategies outperform passive damping. In terms of the maximum deflection, the improvement is now 28 and 25 percent for the centralized and distributed method, respectively. For the passages at speed $v<2 \mathrm{~m} / \mathrm{s}$, we can observe a tendency of a loss of efficiency for both control methods. This is due to the fact that, for low travel velocities, the system operates quasi-statically, and the effect of the two-sided lever (see Figure 5) becomes negligible. The trajectories of the passive and controlled systems at the speed $v=0.5 \mathrm{~m} / \mathrm{s}$ are almost 


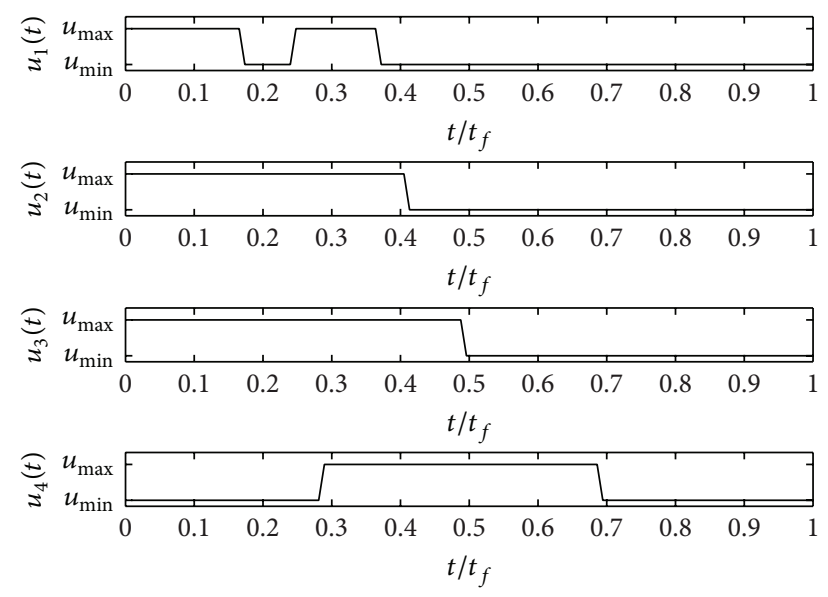

Figure 12: Controls of the designed distributed system in the case of $v=4 \mathrm{~m} / \mathrm{s}$.
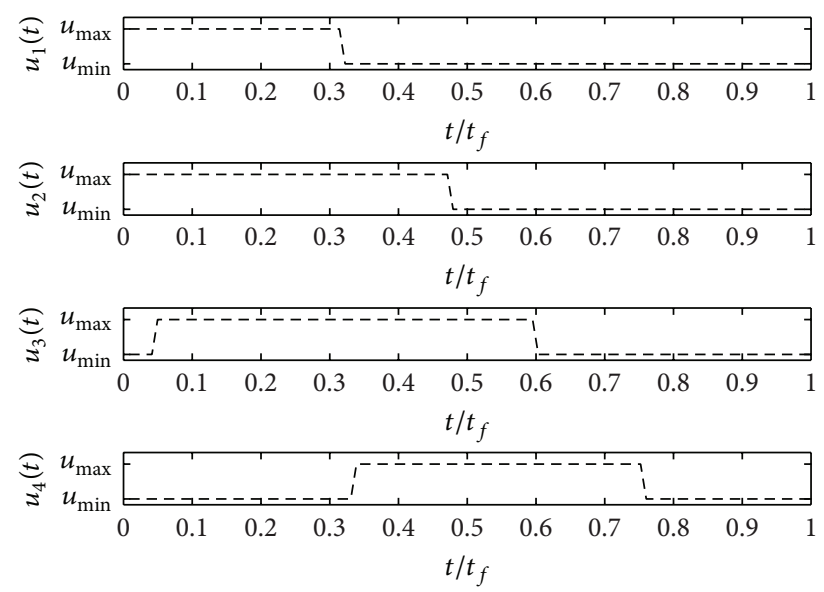

FIGURE 13: Optimal centralized controls in the case of $v=4 \mathrm{~m} / \mathrm{s}$.

TABLE 2: The values of the metric $J_{1}$ normalized to the passive case.

\begin{tabular}{lccc}
\hline \multirow{2}{*}{ Passage speed $[\mathrm{m} / \mathrm{s}]$} & Passive damping & \multicolumn{2}{c}{ Controlled damping } \\
& & Centralized & Distributed \\
\hline 1 & 1.000 & 0.460 & 0.501 \\
2 & 1.000 & 0.351 & 0.389 \\
4 & 1.000 & 0.228 & 0.265 \\
\hline
\end{tabular}

identical. On the other hand, in the case of fast passages, we can notice very high efficiency. The experimental stand does not enable reaching $v=10 \mathrm{~m} / \mathrm{s}$, but the numerical simulation shows that at this speed, by using the distributed method, the maximum deflection can be reduced by 45 percent.

The set of values for the metrics defined in (38) is presented in Tables $2-5$. We can clearly observe the correlation between all metrics. In particular, the maximum deflection of the guideway $J_{2}$ is almost identical with the maximum deflection on the trajectory of the traveling carriage $J_{4}$. The total deflections represented by $J_{1}$ and $J_{3}$ also coincide. Thus, for the optimal selection of the parameter $\alpha$, we can pretty much rely on any one of the proposed metrics.

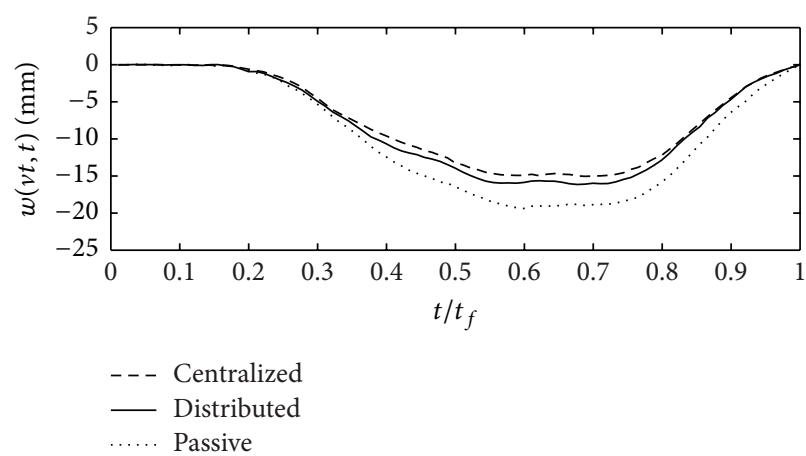

FIGURE 14: Moving load trajectories in the case of $v=2 \mathrm{~m} / \mathrm{s}$.

TABLE 3: The values of the metric $J_{2}$ normalized to the passive case.

\begin{tabular}{lccc}
\hline \multirow{2}{*}{ Passage speed $[\mathrm{m} / \mathrm{s}]$} & Passive damping & \multicolumn{2}{c}{ Controlled damping } \\
& & Centralized & Distributed \\
\hline 1 & 1.000 & 0.808 & 0.812 \\
2 & 1.000 & 0.734 & 0.778 \\
4 & 1.000 & 0.642 & 0.691 \\
\hline
\end{tabular}

TABLE 4: The values of the metric $J_{3}$ normalized to the passive case.

\begin{tabular}{lccc}
\hline \multirow{2}{*}{ Passage speed $[\mathrm{m} / \mathrm{s}]$} & Passive damping & \multicolumn{2}{c}{ Controlled damping } \\
& & Centralized & Distributed \\
\hline 1 & 1.000 & 0.539 & 0.594 \\
2 & 1.000 & 0.388 & 0.407 \\
4 & 1.000 & 0.319 & 0.342 \\
\hline
\end{tabular}

TABLE 5: The values of the metric $J_{4}$ normalized to the passive case.

\begin{tabular}{lccc}
\hline \multirow{2}{*}{ Passage speed [m/s] } & Passive damping & \multicolumn{2}{c}{ Controlled damping } \\
& & Centralized & Distributed \\
\hline 1 & 1.000 & 0.812 & 0.837 \\
2 & 1.000 & 0.719 & 0.755 \\
4 & 1.000 & 0.658 & 0.714 \\
\hline
\end{tabular}

As was to be expected, both control methods outperform the passive case, and the optimal centralized method exhibits the best performance for all metrics at all traveling speeds. In the comparison between the centralized and the proposed distributed controller, we can see an average loss of performance of 4 percent when applying the distributed method. Taking into account that the control law is heuristic and is realized through a very practical distributed architecture, this result is fully satisfactory. Note that the performance of both methods is comparable for passages at all speeds, which supports the assertion of the robustness of the designed controller.

\section{Conclusion}

In this paper, a distributed method to control the vibration of structures subjected to traveling loads has been presented. The method is based on the optimal approach proposed by the author in $[15,16]$. Thanks to a state feedback control 
law and a distributed architecture, the new controller is robust and is easy to implement. The performance has been tested experimentally for a wide range of travel speeds. The efficiency of the new controller is comparable with that obtained with the optimal strategies. The presented solution is practical and is directed to a wide range of applications in transportation and robotic technology. In future research, the author will address the problem of obtaining a distributed control design to suppress the vibration of structures represented by plates and membranes.

\section{Conflict of Interests}

The author declares that there is no conflict of interests regarding the publication of this paper.

\section{Acknowledgment}

The research has received funding from the Foundation for Polish Science under Grant agreement HOMING PLUS/2013-8/11.

\section{References}

[1] T. Frischgesel, K. Popp, H. Reckmann, and O. Schütte, "Regelung eines elastischen Fahrwegs inter Verwendung eines variablen Beobachters," Technische Mechanik, vol. 18, pp. 44-55, 1998.

[2] A. Baz, "Dynamic boundary control of beams using active constrained layer damping," Mechanical Systems and Signal Processing, vol. 11, no. 6, pp. 811-825, 1997.

[3] M. Pietrzakowski, "Active damping of beams by piezoelectric system: effects of bonding layer properties," International Journal of Solids and Structures, vol. 38, no. 44-45, pp. 7885-7897, 2001.

[4] C. A. Tan and S. Ying, "Active wave control of the axially moving string," Journal of Sound and Vibration, vol. 236, no. 5, pp. 861880, 2000.

[5] T. Szmidt and P. Przybylowicz, "Critical flow velocity in a pipe with electromagnetic actuators," Journal of Theoretical and Applied Mechanics, vol. 51, pp. 487-496, 2013.

[6] P. Flont and J. Holnicki-Szulc, "Adaptive railway truck with improved dynamic response," in Proceedings of the 2nd World Congress of Structural and Multidisciplinary Optimization (WCSMO-2 '97), vol. 4, Zakopane, Poland, May 1997.

[7] D. Stancioiu and H. Ouyang, "Optimal vibration control of beams subjected to a mass moving at constant speed," Journal of Vibration and Control, pp. 1-16, 2014.

[8] D. Karnopp, M. J. Crosby, and R. A. Harwood, "Vibration control using semiactive force generators," ASME Journal of Engineering for Industry, vol. 96, no. 2, pp. 619-626, 1974.

[9] Y. Chen, C. A. Tan, L. A. Bergman, and T. C. Tsao, "Smart suspension systems for bridge-friendly vehicles," in Smart Structures and Materials 2002: Smart Systems for Bridges, Structures, and Highways, vol. 4696 of Proceedings of SPIE, pp. 52-61, San Diego, Calif, USA, March 2002.

[10] D. Giraldo and S. J. Dyke, "Control of an elastic continuum when traversed by a moving oscillator," Structural Control and Health Monitoring, vol. 14, no. 2, pp. 197-217, 2007.
[11] A. Ruangrassamee and K. Kawashima, "Control of nonlinear bridge response with pounding effect by variable dampers," Engineering Structures, vol. 25, no. 5, pp. 593-606, 2003.

[12] K. Yoshida and T. Fujio, "Semi-active base isolation for a building structure," International Journal of Computer Applications in Technology, vol. 13, no. 1, pp. 52-58, 2000.

[13] F. Zhou, P. Tan, W. Yan, and L. Wei, "Theoretical and experimental research on a new system of semi-active structural control with variable stiffness and damping," Earthquake Engineering and Engineering Vibration, vol. 1, no. 1, pp. 130-135, 2002.

[14] C. I. Bajer and R. Bogacz, "Active control of beams under moving load," Journal of Theoretical and Applied Mechanics, vol. 38, pp. 523-530, 2000.

[15] D. Pisarski and C. I. Bajer, "Semi-active control of 1D continuum vibrations under a travelling load," Journal of Sound and Vibration, vol. 329, no. 2, pp. 140-149, 2010.

[16] D. Pisarski and C. I. Bajer, "Smart suspension system for linear guideways," Journal of Intelligent and Robotic Systems: Theory and Applications, vol. 62, no. 3-4, pp. 451-466, 2011.

[17] B. Dyniewicz and C. I. Bajer, "New feature of the solution of a Timoshenko beam carrying the moving mass particle," Archives of Mechanics, vol. 62, no. 5, pp. 327-341, 2010.

[18] C. I. Bajer and B. Dyniewicz, "Numerical modelling of structure vibrations under inertial moving load," Archive of Applied Mechanics, vol. 79, no. 6-7, pp. 499-508, 2009.

[19] D. Pisarski, Semi-active control system for trajectory optimization of a moving load on an elastic continuum [Ph.D. thesis], 2011.

[20] B. Dyniewicz, D. Pisarski, and R. Konowrocki, "Semi-active control of track subjected to an inertial moving load," Vibrations in Physical Systems, vol. 25, pp. 147-152, 2012.

[21] R. O. Saber and R. M. Murray, "Consensus protocols for networks of dynamic agents," in Proceedings of the American Control Conference, pp. 951-956, June 2003.

[22] R. A. Horn and C. R. Johnson, Matrix Analysis, Cambridge University Press, Cambridge, UK, 1987.

[23] R. Olfati-Saber and R. M. Murray, "Consensus problems in networks of agents with switching topology and time-delays," IEEE Transactions on Automatic Control, vol. 49, no. 9, pp. 1520 1533, 2004. 


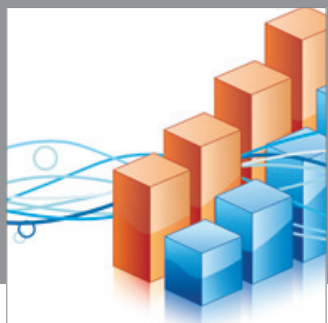

Advances in

Operations Research

mansans

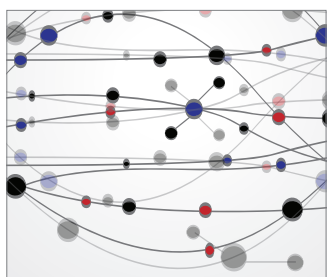

The Scientific World Journal
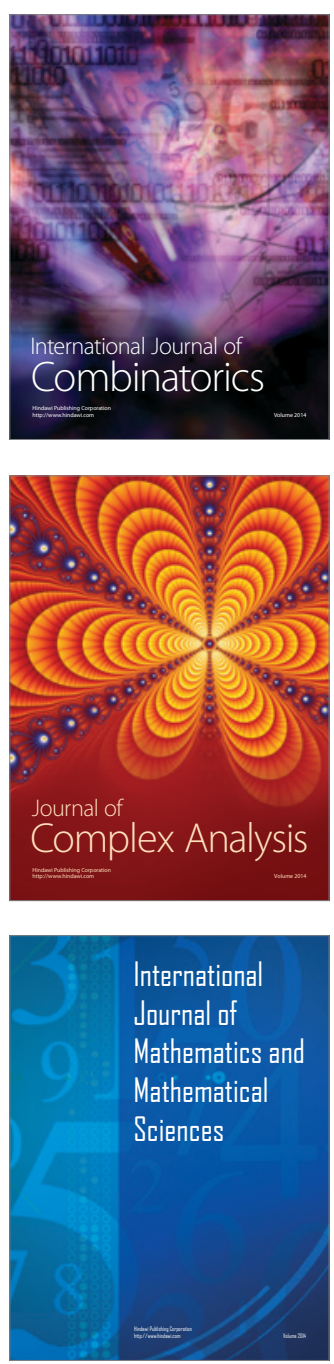


Submit your manuscripts at http://www.hindawi.com
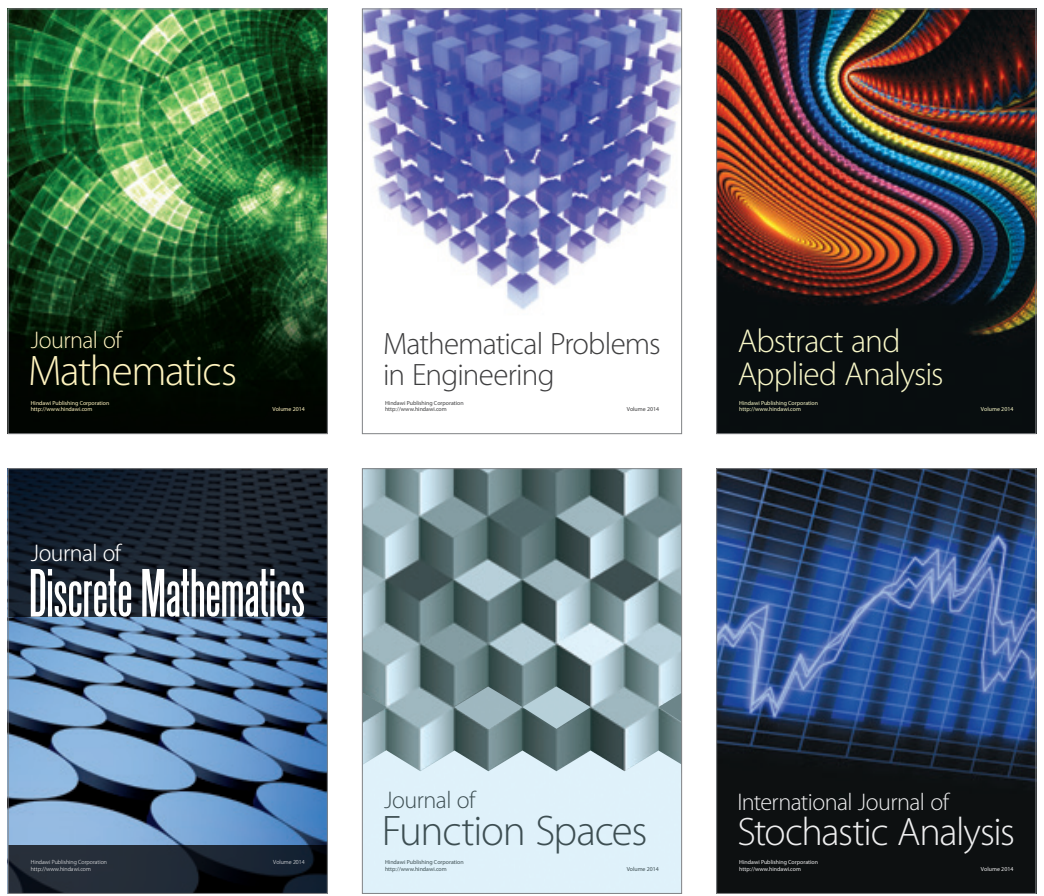

Journal of

Function Spaces

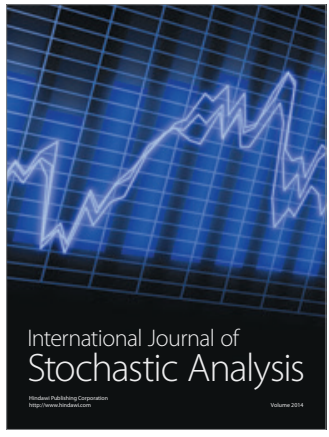

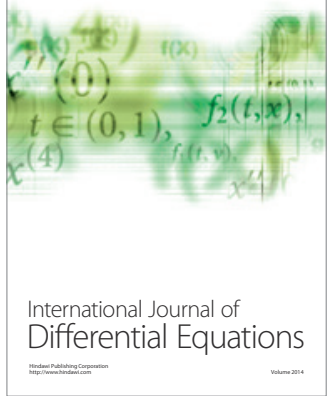
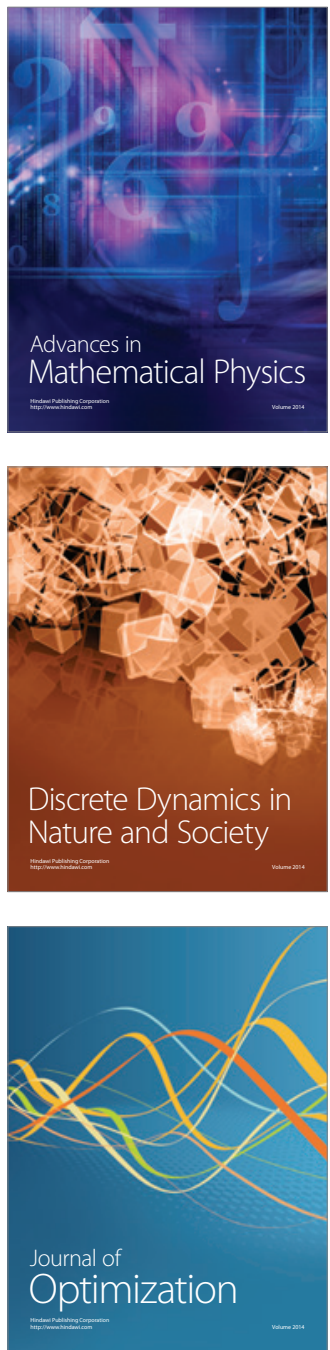